Théologiques

Théologiques

\title{
La théologie au Brésil et le mouvement altermondialiste
}

\section{Ivone Gebara}

Volume 18, numéro 1, 2010

Les Églises chrétiennes et la mouvance altermondialiste

URI : https://id.erudit.org/iderudit/1003547ar

DOI : https://doi.org/10.7202/1003547ar

Aller au sommaire du numéro

\section{Éditeur(s)}

Faculté de théologie et de sciences des religions, Université de Montréal

\section{ISSN}

1188-7109 (imprimé)

1492-1413 (numérique)

Découvrir la revue

\section{Citer cet article}

Gebara, I. (2010). La théologie au Brésil et le mouvement altermondialiste. Théologiques, 18(1), 93-114. https://doi.org/10.7202/1003547ar

\section{Résumé de l'article}

Cet article présente de façon critique quelques réflexions sur le mouvement altermondialiste et la théologie au Brésil. Débutant par un bref rappel historique sur le changement du rôle des religions et des théologies, l'auteur présente les théologies comme des pouvoirs qui veulent influencer les comportements des citoyens et des citoyennes. Néanmoins, ces pouvoirs sont plus complexes que du temps des utopies libertaires des années 1960-1990, notamment parce que des intérêts politiques, économiques et idéologiques se dissimulent souvent sous le voile de la religion. La théologie féministe est aussi présentée comme un pouvoir souhaitant changer les rapports inégaux qui caractérisent les institutions culturelles, ainsi que les représentations religieuses qui privilégient un christianisme à l'aspect masculin. L'article rappelle enfin combien les nouvelles thématiques de la théologie au Brésil sont marquées par les problèmes actuels des sciences sociales et de l'écologie, au risque d'oublier à nouveau la situation des femmes, souvent considérées comme des « sujets " subordonnés à d'autres. 


\section{La théologie au Brésil et le mouvement altermondialiste}

Ivone GEBARA*

\section{Introduction: le changement du monde}

L'être humain est toujours en mouvement dans son histoire personnelle et collective. Tout change, même si nous conservons certaines choses semblables à ce qui a été vécu avant ? et ici je pense particulièrement à l'inévitable rapport entre les différents espaces et temps. Tout change, c'est une vieille constatation. Les sciences, les techniques, les façons de nous comprendre et de nous exprimer, ainsi que les manières de vivre nos conflits et de formuler notre foi ou nos espérances. Tout change et cette mobilité de toutes les choses se montre aussi dans les façons de comprendre les injustices personnelles ou sociales et d'agir sur elles. À chaque époque, nous portons un regard sur nous-mêmes et sur les autres, proches ou lointains. Ainsi, nous vivons de continuelles métamorphoses dans nos relations, même si souvent nous employons les mêmes mots. Néanmoins, ces mots ne possèdent pas la même histoire qu'avant et leur contenu change aussi. À titre d'exemple, afin de montrer combien la mutation est présente, on a vu naître depuis le dernier siècle des histoires sur la vie privée, sur l'amour, sur le sens de la justice, sur l'espérance, etc.

Nous arrivons au XXI ${ }^{\mathrm{e}}$ siècle. Qu'a-t-il de neuf dans notre vieux monde? Qu'a-t-il changé pour les mouvements sociaux et les théologies?

* Ivone Gebara est, depuis vingt ans, philosophe et théologienne nomade. Elle est régulièrement invitée par différents groupes et dans des universités pour enseigner et partager ses réflexions, qui ont pour horizon la philosophie et la théologie féministe de la libération. Son dernier livre (2010) s'intitule Que é saudade. Il est paru aux éditions Brasiliense et touche certaines questions relatives à l'expérience humaine de la nostalgie et à l'absence des choses et des personnes aimées. Elle prépare actuellement un autre livre, sur les rêves et les cauchemars des femmes chrétiennes, qui est attendu aux éditions Oltri (Italie). 
Je me permets de faire, sans être spécialiste, un rapide survol de certaines importantes questions pour situer les nouvelles coordonnées de l'Amérique latine dans le contexte mondial. Entre autres choses importantes, au XXI ${ }^{\mathrm{e}}$ siècle, on commence à parler de plus en plus des mouvements altermondialistes, afin de marquer la nouveauté spécifique par rapport aux différentes luttes et mouvements sociaux des XIX et $\mathrm{XX}^{\mathrm{e}}$ siècles. La nouveauté est apportée par le processus croissant de globalisation de la culture et de l'économie, dans un monde où tout peut être communiqué et, d'une certaine façon, vécu en temps réel. Culture et économie sont dorénavant vues comme des processus plus intimement interdépendants. Néanmoins, l'inégalité poursuit aussi son chemin et se montre sous différentes formes, soit à un niveau local, soit à un niveau international.

L'altermondialisme semble être une réaction à un monde qui est devenu une sorte de grand village, de par la similarité des formes d'organisation économique et la similitude des formes d'organisation culturelle, à la suite de l'énorme capacité de communication et de mobilité que nous avons acquise. Dans cette logique, les formes de domination et d'exclusion sociale ne viennent plus directement des empires coloniaux ou des maîtres particuliers comme les maîtres féodaux par rapport à leurs esclaves ou les patrons par rapport à leurs ouvriers. De plus, ce ne sont plus les grandes puissances qui ont triomphé lors de la Deuxième Guerre mondiale qui déterminent les règles du jeu économique, même si leur pouvoir perdure encore notablement. Dans ce contexte, il ne faut pas oublier la force active de l'Empire américain qui, malgré la crise économique mondiale qu'il a pu déclencher, continue d'avoir une force militaire et économique énorme. Néanmoins, le scénario mondial a changé et ce sont maintenant les grandes corporations qui ont pris la place des maîtres et des patrons de l'économie mondiale - dans un sens, elles sont devenues les nouveaux oppresseurs des pauvres, même si, à certains égards, elles ont fourni du travail et permis le développement de certaines régions. Comme on le disait dans les années 1970 et 1980 en Amérique latine, il faut reconnaître l'ennemi pour le combattre. Et les mouvements sociaux arrivaient à identifier un ennemi souvent considéré comme complice de l'Empire américain et des forces capitalistes. Ce genre d'analyse se répandait aussi dans les mouvements de libération des Églises qui, à l'époque, parlaient de l'importance d'avoir des outils scientifiques d'analyse sociale pour bien faire de la théologie.

Actuellement, alors que la perception et la réflexion sociale sont des activités piégées, on connaît peu le visage des responsables du pouvoir. Ils 
se cachent derrière des structures organisationnelles complexes et ont plusieurs échelons de responsabilité dans les différents pays. Les gouvernements des nations sont maintenant soumis aux grandes corporations et, souvent, les autorités politiques et militaires leur obéissent. Les réunions à Davos (Suisse) démontrent bien la soumission à leurs maîtres et seigneurs des chefs d'État les plus riches au monde. Souvent, un chef d'État n'est qu'un figurant de moindre importance dans le jeu des forces économiques.

Cette situation nouvelle et complexe, surtout par rapport au dernier siècle, a permis le surgissement de nouvelles problématiques et de formes d'organisations qui font face à de nouveaux défis. Notre capacité humaine à nous rendre mutuellement esclaves et seigneurs, ainsi que de faire du profit, se présente toujours sous de nouvelles formes. Et c'est bien pour cela que des nouvelles consignes sont proposées. "Penser globalement et agir localement », «regrouper des formes plurielles de forces sociales », «bâtir des réseaux entre les groupes et les peuples ", "élargir notre combat aux écosystèmes comme parties essentielles de notre vie» sont quelques orientations prises par différents groupes. Dans ces consignes sont inclus les mouvements des femmes, les mouvements écologiques, les mouvements pour le pluralisme de l'orientation sexuelle, les mouvements pour l'égalité entre les ethnies et plusieurs autres. Le même combat est diversifié en plusieurs fronts de lutte pour la dignité de l'humanité et de toute la planète.

De nouveaux problèmes et des situations déplorables qui, jusqu'ici, restaient cachés, apparaissent à la lumière du jour. Ils sont diffusés sur les grandes chaînes nationales et internationales, avec leurs idéologies propres. Ou encore par la petite presse alternative qui nous invite à penser autrement notre monde. En fait, nous n'avons jamais eu autant de groupes différents dans leur action et leur pensée utilisant les moyens que la science technologique met à notre disposition. Aujourd'hui, les choses bougent, mais sans correspondre aux attentes du passé et sans la certitude d'arriver à l'objectif recherché par chaque groupe. On se mobilise, de même que l'on s'assoit devant son ordinateur pour participer aux différentes informations et luttes sociales. Une nouvelle manière d'interaction nous donne l'impression d'être au rendez-vous des grandes décisions sur la vie humaine et la planète. Mais aucune certitude ne nous est donnée.

Le mouvement continuel des choses, à la vitesse des grands ordinateurs, nourrit nos différents combats, mais n'assure plus une visée commune comme au temps des grandes utopies sociales vécues au siècle dernier. À cette époque, nous avions une vision de base, un projet historique à pour- 
suivre à partir des différents groupes sociaux. Nous avions des étapes et des évaluations de notre activité à faire entre nous, c'est-à-dire entre différents groupes d'activistes sociaux. Nous percevions plus clairement nos ennemis et leurs stratégies. Maintenant, nos combats doivent produire des résultats presque dans l'immédiat, comme si notre avenir se réduisait au présent. Il y a une nouvelle manière de sentir et de vivre le temps. La conscience que la planète va mourir nous donne une espèce d'immédiateté par rapport aux fruits de nos actions et ébranle les anciennes croyances religieuses. C'est maintenant ou jamais, disent les gens, même si on connaît les contraintes du présent et les difficultés pour arriver là où notre désir nous pousse.

Même notre désir est devenu plus obscur qu'autrefois. Nous avons du mal à savoir ce qui est le mieux pour nous en tant que collectivité. Les idéologies politiques et les religions ne sont plus capables de faire face à l'intense mobilité de notre désir et aux défis qu'elle impose. De manière particulière, les problèmes du christianisme dans son vécu quotidien et dans sa catéchèse semblent souvent absents de cet univers technologique et consumériste. C'est comme si la répétition du même discours religieux nous ouvrait à un monde enchanté, presque à un monde de fées, si différent du présent, un monde qui s'articule difficilement aux nouveaux langages et aux valeurs de la réalité mobile d'aujourd'hui. Pour être plus exact, le monde de la religion utilise la technologie tout en gardant les vieux contenus, comme s'il existait une "vérité " que la technologie ne pouvait pas toucher. Parfois, nous avons l'impression que le monde de la religion est distinct du monde ordinaire et, parfois, il y a comme une communion entre eux.

La globalisation nous met à la merci d'un contexte national et international beaucoup plus complexe. Nos analyses et nos solidarités changent souvent. La vitesse des événements transforme très vite le présent en passé. Et l'écart entre les générations semble s'accentuer. C'est comme si les générations des années 1940 ou 1950, qui ont mené plusieurs combats en Amérique latine, se sentaient plus ou moins perdues devant l'avancée de la technologie qui n'a plus besoin des mêmes utopies, ni des discours éthiques du passé, ni même des discours théologiques libérationnistes.

Dans la même ligne, il faut aussi rappeler que dans différents pays d'Amérique latine nous avons vu, ces dix dernières années, la victoire de gouvernements populaires à différentes échelles de l'organisation politique et sociale. Cela a certainement changé la direction des luttes politiques. Maintenant, quelques-uns de ceux qui représentaient la force des pauvres 
sont au pouvoir, mais les problèmes n'ont pas encore trouvé la solution attendue.

À partir de ce contexte en mutation rapide, nous continuons à nous poser des questions: Que voulons-nous maintenant? Que veulent les théologiens? Que veulent aussi les nouvelles générations par rapport à la théologie ? Comment comprendre à nouveau aujourd'hui la question fondamentale de la foi chrétienne: qui est mon prochain, celui et celle qui sont là en attente de mon amour? Avec quelle réciprocité ou quelle gratuité faut-il les aimer? Dieu est-il/elle Amour ? Les multiples soupçons guettent nos certitudes du passé et du présent.

\section{En quoi la théologie concerne-t-elle le monde d'aujourd'hui ?}

Depuis la deuxième moitié du $\mathrm{XX}^{\mathrm{e}}$ siècle, la théologie a pris un tournant de plus en plus pluriel, tout en gardant les principaux contenus traditionnels. Elle les a réinterprétés selon différentes références, contextes historiques ou situations. Elle s'est ouverte aux nouvelles approches bibliques et aux sciences sociales, ce qui lui a donné une dimension moins idéaliste et dogmatique, tout en étant plus contextuelle et dialogique. On dira qu'une richesse extraordinaire est apparue à la lumière du jour, et qu'elle est aussi arrivée dans les différents pays du tiers-monde.

Les théologies de la libération ont eu des développements particuliers dans les différents continents. L'option pour les pauvres comme option de foi a été la clef de voûte de cette théologie, la source des nouvelles théologies et des alliances avec les différents mouvements sociaux pour instaurer des relations de justice et d'équité. Au Brésil, par exemple, des théologiens de la libération ont appuyé la fondation du Parti des Travailleurs avec l'ancien syndicaliste Lula, devenu ensuite le président de la République. De même, différentes organisations pour la réforme agraire, pour la défense des Autochtones et pour le droit à l'habitation ont pu compter sur l'appui des Églises et surtout des théologiens de la libération. On ne pouvait plus parler de Dieu comme Amour et Justice sans essayer de faire vivre ces valeurs dans nos relations sociales et politiques. Le Ciel ou le Royaume de Dieu devait commencer à être vécu sur terre. Ces idéaux ne concernaient plus un au-delà de l'histoire, il fallait au contraire goûter déjà sur terre les valeurs qui orientent la vie. Tout cela est bien connu et pourra être lu dans les publications des années 1970 à 2000. Néanmoins, même si tout cela est vrai, il faut aussi dire que la formation religieuse, la catéchèse dans les différentes paroisses ou chapelles du continent n'ont pas été influencées par les 
nouveaux contenus. Les gens simples lisent peu et surtout peu de théologie, malgré quelques efforts de diffusion de textes dans un langage plus simple et direct. Ce processus libérationniste a été plutôt lié aux mouvements sociaux et, pour la majorité des gens, il n'a pas réellement changé la compréhension hiérarchique du christianisme. Rappeler ce phénomène est de la plus haute importance pour comprendre les difficultés que nous avons actuellement à dépasser une mentalité religieuse magique qui se fait encore très présente aujourd'hui en différents lieux.

Dans cette perspective, quel a été le sens de la richesse théologique évoquée et que se passe-t-il dans le présent? Quelles influences ces théologies ont-elles eues sur la vie des fidèles pour que se forme ce moment nouveau qu'est l'aujourd'hui? Et aujourd'hui, quelles sont les grandes lignes de l'influence du christianisme dans nos différents milieux?

Je ne saurai précisément répondre à ces questions, mais, même si cela surprendra mes lecteurs et mes lectrices, j'ébauche progressivement une nouvelle réflexion proposant de voir la théologie comme une idéologie du pouvoir religieux au niveau des institutions sociales du christianisme. Sans doute, elle est plus que cela, mais j'ai besoin d'un point de départ limité pour faire apparaître mon propos et poursuivre ma réflexion. La théologie est une idéologie plurielle du pouvoir religieux qui accompagne les différentes forces présentes dans nos sociétés. Je ne prends pas ici le mot idéologie dans sa formulation négative, mais comme un système d'idées qui vise à influencer des comportements et orienter des actions. Dans la constitution même du pouvoir religieux, il y a différents échelons, c'est-à-dire qu'il y a une participation inégale des différents groupes de fidèles ainsi que des membres de la hiérarchie des Églises.

Nous savons que le pouvoir religieux n'est pas homogène et qu'il suit les différents courants qui sont présents dans la société. Il n'a pas d'autonomie totale par rapport aux différentes forces qui agissent dans la société. Avant qu'un citoyen ne soit clerc ou théologien, il est un citoyen qui se positionne par rapport aux différentes questions de son milieu et de son temps. Ce qui est premier, c'est l'option de société, le modèle de famille, la direction politique qu'on assume dans le clair-obscur de nos choix, la place qu'on a ou qu'on cherche à avoir. Ensuite, ou, peut-être en même temps, on s'habille des pensées théologiques, croyant qu'elles sont les plus importantes et les plus décisives dans les choix que nous avons faits. On habille nos choix politiques avec les vêtements de la religion et nos discours avec les concepts courants de la théologie, croyant à la primauté de la théologie. 
Souvent, les personnes impliquées en religion ont l'impression que la lecture religieuse de la vie est première et que c'est à partir de cette lecture qu'elles ont pris telle ou telle orientation, embrassé telle ou telle cause. C'est comme si leurs chemins étaient établis d'avance par leur Dieu et que les orientations sociales et politiques découlaient de ce choix premier. En réalité, il n’y a pas moyen de déterminer ce qui est premier puisqu'ici nous sommes sur le terrain de la subjectivité, des histoires personnelles avec leurs richesses et leurs pièges.

Quoi qu'il en soit, la théologie reste une politique, un pouvoir capable d'influencer les comportements des personnes, ainsi que les institutions sociales et politiques. Et, j'insiste, elle est une politique dans la mesure où elle veut aussi intervenir dans le fonctionnement de la polis, à travers les moyens d'action de ses adeptes. Ce pouvoir cherche à augmenter le nombre de ses fidèles, sans prétendre - à première vue — avoir immédiatement la direction des lieux de décision politique et économique. Dans le quotidien, on peut observer que ce pouvoir cherche à avoir de l'influence sur les mœurs des croyants, laissant entendre croire que leurs positions éthiques viennent directement de leur Dieu — que l'on pense, par exemple, à toutes les discussions autour de la question de la procréation, de l'avortement, des cellules-souches, du mariage des homosexuels ou de l'adoption des enfants par des couples homosexuels, qui sont toutes des nouvelles questions, par rapport à celles des années 1980, qui concernaient plus l'éthique dans la politique et l'économie. L'intervention de la foi religieuse dans la sphère de décision publique a été et est encore énorme en Amérique latine, même avec de nouveaux acteurs. C'est bien pour cela qu'il ne faut pas oublier qu'il s'agit d'un pouvoir qui se présente comme spirituel ou comme venant d' "en haut », mais qui agit sur la matérialité de la vie, sur la décision des corps réels faisant face à un bon nombre de questions urgentes.

La représentativité officielle de ce pouvoir est la propriété d'un clergé masculin. Il faut toutefois dire qu'en plus du pouvoir du clergé, il y a le pouvoir, par exemple, des grandes corporations catholiques, souvent laïques, qui agissent dans les différents milieux économiques et politiques. Personne n'ignore la force politique de mouvements comme l'Opus Dei, les Hérauts de l'Évangile et bien d'autres. Ils sont des banquiers, des chefs d'entreprise, des cadres catholiques qui se présentent comme des défenseurs des valeurs éternelles du christianisme. Et ici aussi, il y a la difficulté d'un pouvoir religieux qui se montre de façon claire chez quelques-uns et de façon nébuleuse chez d'autres. Souvent, on attribue au clergé les déci- 
sions doctrinales, mais c'est la permanence de certains choix politiques et économiques qui finit par maintenir vivantes certaines doctrines, empêchant l'apparition de nouvelles questions ou positions.

Le bon sens nous dit que rien n'est clair en politique ni en religion. Tout est le fruit d'interprétations mouvantes qui finissent souvent en conflit les unes avec les autres. Aujourd'hui, le pouvoir religieux clérical n'est bien souvent qu'une simple apparence. Le bon curé de village, avec son autorité directe sur la vie des fidèles, n'existe presque plus. Le monde a changé et même le pouvoir religieux est entre les mains des nouveaux représentants de l'empereur Constantin, toujours prêts à accorder certaines faveurs ou à soutenir des œuvres sociales au prix de l'appui clérical.

Je continue d'affirmer que la religion est un pouvoir par-dessus d'autres pouvoirs ou parmi d'autres pouvoirs. Il se croit soutenu, d'un côté, par Dieu et, de l'autre, par le peuple croyant. Dans cette situation existentielle, les fidèles de la religion croient travailler pour le bien des gens, pour leur salut historique, voire au-delà de l'histoire. Le paradoxe continue d'être présent: on avance, mais on retrouve régulièrement des obstacles au milieu du chemin! Comment rendre claire cette construction concrète du pouvoir historique avec les fondements abstraits du pouvoir religieux?

Particulièrement dans le contexte des théologies de la libération, où l'option pour les pauvres et les démunis a été capitale, le jeu entre la volonté des théologiens, celle des activistes ecclésiaux et celle de leur Dieu a été fortement présent dans un passé récent. Les théologiens étaient convaincus d'exprimer la volonté de Dieu et ils arrivaient à la rendre présente au moyen de différentes lectures matérialistes ou spirituelles de la Bible. Dans cette perspective, parler de théologie, c'était parler de quelque chose de bien et de bon pour les gens. Parler des théologiens, et surtout de théologiens de la libération, c'était parler des gens du côté du bien, de la vérité et de la justice, comme si la balance du bien et du mal penchait très peu du côté du plateau du mal. Les théologiens, les pasteurs, les prêtres sont des gens auxquels il faut faire confiance, puisqu'ils ont une qualité morale reconnue et qu'ils sont des envoyés de Dieu. Ils représentent, d'une certaine façon, la réserve éthique de la société, c'est-à-dire des gens incorruptibles qui représentent la possibilité de croire à la sincérité humaine et à la recherche de la vérité. En plus de leurs connaissances relativement vastes, ils imaginent que leur savoir est "garanti », puisqu'ils se présentent, vis-à-vis du peuple, comme étant du côté de Dieu. C'est bien pour cela que n'importe quel scandale ou n'importe quel écart de comportement possède encore un effet dramatique lorsque 
cela vient du clergé ou d'un religieux. Nos sentiments et nos analyses sont toujours marqués par nos préjugés.

Dieu, pour sa part, veut notre bien, de même que les théologiens et les pasteurs. J'utilise ici le masculin pour exprimer ce pouvoir, tout en étant consciente des incursions du féminin dans ce domaine. Néanmoins, il y a sans aucun doute une primauté du pouvoir masculin dans la tradition chrétienne, qui est encore présente aujourd'hui.

Une des caractéristiques du pouvoir «divin » de la religion réside dans le fait qu'il se place toujours en juge de la société. Il a toujours raison, même si, selon des analyses, il a tort. La société avec ses multiples problèmes n'obtient jamais la totale approbation divine. Si cela arrivait, ce serait peut-être la fin des Églises et des théologiens, ce serait aussi la fin des prêcheurs publics. Leur fonction, qui consiste à se placer en conscience morale, à juger et à exposer aux gens les voies de Dieu, serait terminée. Il faudrait leur découvrir une autre fonction ou les transformer en autre chose.

Mes lectrices et lecteurs doivent se demander où je souhaite en venir avec ces idées qui semblent nous écarter du sujet proposé. Elles et ils sont probablement surpris par l'ironie et l'humour dont je fais preuve, par rapport à des choses aussi sérieuses que la théologie. Mais l'humour aide à penser et il nous replace face aux contradictions de notre vécu. Sur cette pensée, je reprends le fil de ma réflexion, qui consiste à considérer aujourd'hui la théologie comme pouvoir et comme politique. Quand le pouvoir du monde devient un pouvoir globalisé, un pouvoir dans les mains des grandes corporations, nous pouvons nous demander ce que devient le pouvoir théologique ou le pouvoir de la religion. Quand la distinction entre le bien et le mal semble se dissiper dans la pluralité du monde, que devient la théologie? Quand les théologiens et les clercs sont soupçonnés de racisme, de misogynie, de malhonnêteté, que devient la théologie?

Le pouvoir de la théologie et de la religion est un des multiples centres de pouvoir présents dans un monde globalisé. Le pouvoir religieux ne semble plus être un centre d'action dans les institutions religieuses, mais il est présent partout. C'est sa nouvelle forme de manifestation. On ne tient plus compte de lui comme par le passé, comme un pouvoir absolu auquel il fallait se soumettre. Il est en plein milieu des différents conflits sociaux. De même, la séparation entre les pouvoirs religieux et le pouvoir politique national, qui s'est affirmé, depuis le siècle dernier, semble ébranlée par l'invasion du religieux dans toutes les sphères. Le pouvoir religieux n'a plus l'hégémonie de ce qu'on appelle le pouvoir spirituel et il n'a plus un unique 
centre institutionnel. Depuis l'émergence de plusieurs Églises nouvelles et leur présence au sein des instances politiques, à travers certains députés ou sénateurs, la force du religieux est particulièrement présente dans la sphère politique. Au nom de leur foi, ces dernières font valoir leurs positions politiques et leurs choix de société.

Depuis les développements du mouvement œcuménique et du dialogue interreligieux, nous sommes devenus plus souples face à l'approche religieuse des uns et des autres. On insiste sur le mot dialogue, parce que l'on veut sans cesse comprendre les raisons et les choix des autres; on veut respecter les autres pour être nous aussi respectés. On sort du privilège consistant à posséder toute la vérité pour accepter de n'avoir qu'une part de la vérité.

Dans la même perspective, un Forum de théologie qui se tient avant ou après un Forum social est aussi marqué par une décentralisation des pouvoirs. Ceci veut dire que l'importance de la théologie est dorénavant liée à l'importance des discussions sociales et politiques qui se font dans un terrain hors du contrôle des Églises. L'originalité théologique semble vouloir suivre de plus près les questions sociales et économiques qui sont à l'ordre du jour. Or, nous savons que le dernier Forum théologique à Belém (2009) a eu comme thème L'eau, la terre, la théologie en vue d'un autre monde possible. La thématique a été fixée en tenant compte du problème majeur vécu par tous les pays, c'est-à-dire le problème du réchauffement global et de la destruction de l'environnement. Mais quel est le pouvoir de la pensée théologique devant cette question ou d'autres questions partageant la même perspective écologique? La majorité des textes qui ont été diffusés après le Forum théologique ont démontré la pauvreté théologique et pastorale dans laquelle nous sommes aujourd'hui, face à un bon nombre de thèmes. Nous pouvons répéter comme théologiens les alertes des scientifiques, convoquer la communauté à prendre soin de la terre ou de l'environnement. Nous pouvons répéter que l'Évangile nous invite à prendre soin de nous et de la Terre comme des biens de la création. Nous ajoutons notre pouvoir au pouvoir de ceux qui cherchent un équilibre dans l'économie et pour éviter le gaspillage de la Terre. Mais comment comprendre nos doctrines, notre dogmatique, notre façon de lire la Bible, notre tradition théologique, notre catéchèse à partir des nouveaux défis de notre monde? Comment dépasser un certain parallélisme et un certain dogmatisme qui sont souvent présents? Ou encore, comment ces défis peuvent signifier un changement radical de perspective dans les théologies? Je pense que ce travail n'a 
pas été fait, sauf par un petit nombre de personnes, mais qui n'arrivent pas à transmettre ces urgences au vécu des Églises. Que se passe-t-il avec les théologies? Quelle est leur place dans un monde où les repères traditionnels sont remis en question? Je laisse ces questions habiter notre cœur, mais qu'elles soient aussi des aiguillons servant à stimuler notre capacité de penser autrement la cité de l'humanité.

\section{La théologie féministe comme pouvoir}

Je commence cette partie de ma réflexion avec quelques idées tirées de la théologie féministe en Amérique latine. Celle-ci peut aussi être lue à partir de la question du pouvoir et particulièrement le pouvoir des femmes. Je l'adopte pour montrer l'urgence de changements que nécessitent certains contenus théologiques à la lumière des grandes questions de notre temps.

Comme nous le savons, le féminisme est entré de façon plus significative dans la théologie d'Amérique latine à partir des années 1980. La question du pouvoir a toujours été centrale, même si on n'en parlait pas directement dans les Églises. Un pouvoir qui signifiait pour les femmes la conquête des droits pour elles-mêmes, en vue d'un régime de rapports plus égalitaire en société et en Église. Sortir de la tutelle des hommes, du contrôle de leur corps et de leur pensée, sortir des modèles féminins préétablis a signifié, entre autres, l'affirmation de nouvelles identités féminines et de nouvelles formes de relations sociales et de pouvoir. Et ce pouvoir touche aussi le vieux pouvoir de la religion, particulièrement à l'intérieur du christianisme. Néanmoins, les Églises ont été toujours méfiantes vis-à-vis des femmes et surtout du féminisme. Pour le magistère des Églises, l'ascension sociale des femmes à partir du féminisme a signifié un refus de soumission au clergé masculin, ainsi qu'un certain refus des doctrines théologiques qui excluaient les femmes d'une représentativité réelle dans l'Église.

La théologie féministe a essayé de partir des corps des femmes, corps victimes de l'inégalité anthropologique et sociale. Cette inégalité a servi de base à la construction de l'édifice théologique, ainsi qu'au maintien de l'hégémonie du pouvoir masculin dans la culture. C'est à partir des souffrances des femmes et de la conscience de l'inégalité anthropologique que beaucoup de textes féministes ont été écrits. Dénoncer, déconstruire, réinterpréter, resituer, resignifier, refaire ont été des verbes conjugués à l'actif dans les différents apports de la théologie féministe. Néanmoins, le refus d'accueil aux voix féminines en ce domaine a été une constante dans la majo- 
rité des Églises et très particulièrement dans l'Église catholique romaine en Amérique latine.

Pourquoi le féminisme théologique ne s'est-il pas imposé dans l'Église catholique et dans ses institutions comme une théologie parmi d'autres? Pourquoi dans les congrès officiels de théologie, comme dans les réunions des théologiens de la libération, la question du féminisme est-elle souvent mineure et vue comme une concession de la part des organisateurs? Pourquoi a-t-elle souvent provoqué un malaise entre les hommes et parfois aussi entre quelques femmes? Pourquoi la question écologique a pris plus d'intérêt que celle des femmes et pourquoi des questions comme la violence sexuelle vécue et dénoncée surtout dans les situations de guerre ont été presque méprisées? Je pense spécialement à la problématique de la violence sexuelle comme arme de guerre, surtout en Colombie, en Haïti et d'autres pays, qui ne trouve pas d'écho significatif parmi les théologiens. Je pense aussi à différentes questions sociales et politiques qui révèlent un traitement inégal donné aux femmes et qui n’ont pas d'audience dans les milieux religieux.

Je n'ai pas la prétention de donner des réponses à toutes les questions soulevées. Mais j'ose ouvrir quelques pistes de réflexion pour faire apparaître certains aspects de la complexité de la problématique du féminisme en théologie.

Je soupçonne que le refus de la théologie féministe comme problématique majeure est en grande partie dû au défi que le féminisme lance face à l'excès de symbolique masculine dans le christianisme et à la représentation masculine du pouvoir de Dieu. Ceci veut dire que, dans le christianisme, le salut nous vient, au moins symboliquement par les hommes ou par un Homme reconnu et proclamé Dieu. Les hommes sont les symboles les plus éminents du savoir, du pouvoir et du valoir dans les communautés chrétiennes, même si on offre une place spéciale aux femmes comme mères. Ce sont eux qui symbolisent l'amour jusqu'à la mort et le sacrifice rédempteur. Dans cette lancée, les femmes apparaissent souvent comme soumises à ce pouvoir majeur et comme servantes de ce pouvoir, même si la maternité, une composante fondamentale de cette structure, est valorisée à l'extrême.

Le féminisme, surtout depuis la deuxième moitié du $\mathrm{XX}^{\mathrm{e}}$ siècle, a essayé de combattre la reproduction de la domination masculine dans la symbolique politique, culturelle, sociale et religieuse. Mais le féminisme a également ouvert la réflexion sur les rapports intimes entre le privé et le public. Dans ce sens, la question des femmes n'est pas seulement un problème de 
foyer, mais un problème social, politique, économique, culturel et religieux. Et c'est bien cette entrée publique des femmes dans le monde plus vaste qui dérange les théologiens et les clercs. Nous savons combien il est dérangeant d'avoir comme «objet» de justice sa femme, sa mère, sa sœur, sa cousine, sa compagne de travail. Nous savons combien il est difficile de rendre justice à celles qui soutiennent notre travail quotidien. Il ne s'agit pas de justice comme courtoisie, celle que les hommes aiment bien accorder, mais de justice au niveau des droits et de la représentativité sociale. Cela est une des pierres d'achoppement difficilement accueillies par les Églises et les théologies.

Le féminisme en a fini avec la courtoisie formelle des théologiens à l'égard des femmes. Il invite à aller plus loin, à revoir les images masculines du divin, la supériorité indue octroyée au pouvoir masculin. Il invite à penser au mépris du corps féminin et à la violence sexuelle, sujets que les hommes théologiens résistent souvent à faire entrer dans leur réflexion. Ils veulent théologiser à partir des corps victimes d'injustice sociale, mais non à partir des corps sexués qui souffrent des injustices sociales. Ils ne travaillent pas le genre des victimes ni des responsables directs des victimisations.

Souvent, l'officialité des Églises refuse la théologie féministe ou lui donne une place d'appendice; on lui concède un paragraphe en passant dans tel ou tel texte, parce que l'on craint la dénonciation de politiques théologiques ou de politiques ecclésiastiques qui veulent toujours garder l'éminence apparente du masculin. Ce qui constitue l'autre côté du masculin, ce qui est le "proche» du masculin, ce qui est le fondement du masculin, c'est-à-dire le féminin, est éloigné ou méprisé par la théologie. On lui refuse le droit de citoyenneté, on le destine à disparaître devant un sujet majeur comme l'option générique pour les pauvres et, dernièrement, l'option écologique. Il y a toujours plus important que les femmes, plus important que leurs petites questions, le plus important auquel elles doivent se soumettre et dont elles doivent reconnaître la pertinence au risque de perdre la crédibilité vis-à-vis des hommes d'Église. «On en a assez des questions des femmes ", disent plusieurs... «Encore, les questions de violence sexuelle contre les femmes? " Ou encore: "Laissez-nous réfléchir sur le sérieux de la théologie ", disent les autres! Mais, qu'est-ce que «le plus sérieux»? Montrer que le profit du système est en train de détruire la couche d'ozone, que Chinois et Nord-Américains gaspillent de l'énergie, que le réchauffement global grandit et englobe tout le monde, que les glaciers ne vont bien- 
tôt plus exister? Ou dénoncer la violence sexuelle contre les femmes en Colombie, en Afghanistan, en Palestine, en Haïti ? Ou encore, dénoncer la persistance de la faim surtout pour les enfants et les femmes? On ne peut pas nier la pertinence de la situation écologique mondiale, mais on ne peut pas admettre l'oubli des questions concrètes qui touchent ceux et celles qui sont à notre image et ressemblance. On ne peut pas oublier les cris de ceux qui sont plus directement de notre chair, même sachant la complexité de notre constitution comme êtres vivants et de notre responsabilité envers la planète. La colonisation des femmes et du féminin n'est pas encore terminée. Et les chrétiens ont une bonne partie de cette responsabilité, surtout quand on insiste pour garder des formes de domination lues comme obéissance ou désobéissance à la volonté du Père.

La théologienne nord-américaine Mary Hunt a présenté, au Forum théologie et libération à Belém, une conférence intéressante dont le titre était: "Bodies don't Lie: A Feminist Theological Perspective on Embodiment ${ }^{1}$ » ( «Les corps ne mentent pas: une perspective théologique féministe de la corporalité»). Dans cette réflexion, elle souligne la nécessité de ne pas oublier, dans la pensée théologique de l'actualité, l'importance des corps souffrants, des femmes et des enfants victimes de violence dans le quotidien et dans les multiples guerres de notre planète. Les corps ne mentent pas, dit-elle. Ces corps humains sont eux-mêmes l'expression paradigmatique de ce qui se passe dans le corps de la Terre: exploitation de ressources, marginalisation, maladie, ignorance et ainsi de suite. Regarder les corps qui nous côtoient, surtout les corps victimes les uns des autres, devrait nous empêcher de tomber dans les abstractions si communes de la pensée théologique.

Penser la vie exige aussi qu'on puisse faire de l'autocritique. Et c'est dans cette perspective que j'aimerais aussi souligner la nécessité de critiquer certains aspects de la théologie féministe elle-même, dans sa diversité contextuelle. Cette critique a à voir avec un certain esprit, appelé scientifique, qui est entré dans différentes pensées féministes à la suite de l'héritage de notre histoire patriarcale commune. Souvent, nous, théologiennes, utilisons les théories féministes pour comprendre les relations de pouvoir en société, mais souvent ces théories restent valables seulement en milieu académique. Elles ne touchent pas les milieux non universitaires ni les grandes questions brûlantes du monde dans leur contexte précis. Elles s'élaborent

1. Conférence donnée le 24 janvier 2009, à Belém, Brésil. 
et dialoguent entre elles, parfois dans un contexte limité où seulement ceux et celles qui parlent un même langage peuvent se comprendre. Souvent les théologiennes ont appris et reformulé les théories féministes sur le genre ou le patriarcalisme, mais elles ont oublié d'entrer dans la matière de la tradition théologique et de l'ouvrir à partir d'autres points de repère. De même, il faut ajouter que leur travail est souvent refusé par d'autres femmes. Je pense à toute la population de femmes des congrégations religieuses féminines qui rendent un immense service à différents groupes, mais qui, en même temps, reproduisent la même théologie qui, la plupart du temps, ne les libère pas. Plusieurs d'entre elles ont peur de perdre leur vocation et croient que le féminisme peut nuire à leur vocation et à leur fidélité à Dieu.

Souvent, nous, femmes théologiennes, nous avons critiqué l'excès de scientisme des théologiens par rapport à la foi et le réductionnisme de certaines théologies à une science de la religion. Maintenant, parfois, il nous arrive de tomber dans la même tentation et dans les mêmes erreurs. La pensée alternative nous donne un pouvoir, sans doute, mais si ce pouvoir n'est pas utilisé pour alléger les fardeaux des gens et spécialement des femmes, il servira très peu au changement de relations en société. C'est bien pour cela que l'Association des théologiennes et théologiens du tiersmonde a réalisé à Bogotá en septembre dernier une réunion de femmes autour de la question de la violence sexuelle contre les femmes en situation de guerre. Nous savons que depuis longtemps, en Colombie, les femmes sont une cible de guerre de la part des militaires, paramilitaires et drugdealers ou trafiquants.

À partir des victimes et du contexte du pays, nous avons pu nous poser différentes questions théologiques, c'est-à-dire des questions qui ont à voir avec la tradition chrétienne des victimes et de leurs bourreaux. Comment dire Dieu pendant et après une violation sexuelle? Quel sens aura le pardon aux ennemis? Que veut dire être en communion? Avec qui ? Que veut dire aimer mon prochain? Qui est mon prochain? Que faire avec l'enfant planté dans mon corps par la violence?

Le lieu de la théologie n'est que le lieu de la vie courante et si je transforme ce lieu en un lieu de science pure avec un langage plus ou moins hermétique, je m'éloigne de sa force constitutive. Penser théologiquement, c'est penser à partir des événements et à partir de la tradition religieuse vécue par les gens. Penser théologiquement, c'est essayer de vivre toujours à nouveau les contenus de nos croyances les plus profondes, les rendre historiques à partir de notre histoire. Cette tâche reste un défi pour nous. 
Nous approcher des victimes et nous approcher des joies des gens, entrer dans leur vie pour comprendre quelque chose de leur mémoire religieuse, de la tradition de salut collée à leur peau, c'est faire de la théologie autrement. Et c'est aussi retrouver la spécificité de notre mission et de notre magistère.

\section{La pollution dans la théologie chrétienne}

Affirmer la théologie comme pouvoir, c'est l'affirmer aussi comme une pensée contextuelle qui a de l'influence sur des sujets situés dans un temps et un milieu précis. La théologie s'est établie au cours des siècles comme science et comme science sur Dieu et ses désirs pour l'humanité. C'est justement dans la façon dont les désirs de Dieu pour l'humanité se posent, la façon dont les gens ont formulé ses désirs que les jeux des pouvoirs apparaissent le plus clairement. Les mêmes conflits qui nous caractérisent dans d'autres domaines sont présents dans celui domaine de la religion.

J'aimerais limiter ma réflexion à la théologie catholique que je connais un peu mieux. Elle a établi ses vérités comme des dogmes reconnus pour toujours et s'est soumise aux pouvoirs religieux hiérarchiques, responsables des croyances diffusées ou enseignées aux fidèles. C'est cette même théologie, avec sa liaison aux traditions religieuses et aux pouvoirs religieux établis qui change périodiquement d'objet théologique dans la pratique. L'objet théologique dans la pratique ou simplement l'objet pratique de la théologie se montre dans la direction donnée aux interprétations bibliques et théologiques suivant le temps. Le même texte ou le même dogme peut être lu et interprété selon l'esprit et les questions de l'histoire contextuelle. Par exemple, là où on lisait les hommes comme fils de Dieu, on lit maintenant les femmes et les hommes. Là où on parlait de l'enfer dans l'au-delà, on parle de l'enfer ici, et ainsi de suite.

Un des objets de la théologie pratique de l'actualité a à voir avec la destruction planétaire ou avec les problèmes du réchauffement global dû à une destruction écologique sans précédent dans notre histoire. Un nouveau mot est maintenant utilisé, l'écothéologie, afin de marquer l'entrée de ce nouvel enjeu dans la vaste problématique théologique. Les théologiens réunis à Belém en juillet 2009 se sont ouverts à cette question brûlante et se sont présentés aussi au rendez-vous de ceux qui pensent l'histoire. Mais, s'ils ont constaté combien la planète est polluée et détruite par l'intervention des êtres humains, le travail sur la question de la pollution à l'intérieur même de la théologie a été plus limité. Inclure le terme pollution dans 
les concepts théologiques semble étrange et même bizarre. Normalement, on n'attribue pas ce mot à des idées et moins encore à des concepts théologiques. Néanmoins, à partir d'un minimum de réalisme philosophique, nous pouvons dire que tous les concepts humains se réfèrent à des réalités liées à la vie humaine sur la planète. Dans ce sens, même de façon figurée, nous pouvons parler de la pollution des idées et notamment des idées théologiques.

Pollution se réfère à l'acte de salir quelque chose, de la rendre nuisible à la vie, de dévier son objectif de maintenir la vie et remplacer la qualité de vie par la mort au profit de quelques-uns. Dans ce sens, il y a des idées qui, au sens figuratif, sont capables de produire le mépris, la destruction et même la mort pour certains êtres. Par exemple, l'idée de la supériorité raciale a été capable de produire différentes formes de racisme jusqu'au génocide. Nous connaissons toute l'histoire du nazisme et ses variations historiques prolongées jusqu'à nos jours. Nous connaissons également tout le revanchisme sioniste par rapport au monde arabe. Nous savons bien la problématique en Occident entre les Blancs et ceux et celles d'origine africaine, problème encore très présent, malgré le progrès que nous avons vécu. De même, l'idée de la supériorité de l'homme par rapport à la femme a produit différentes distorsions au niveau des droits humains. Et, dans la même ligne, l'excès d'anthropocentrisme a été capable de réduire la terre au profit d'une élite. Nous pouvons nommer encore tant d'autres problèmes... C'est dans ce sens précis que je parle aussi de pollution dans la théologie, c'est-à-dire d'une capacité de produire la mort ou d'être complice de la mort par le maintien de certains concepts, formes de pensée ou traditions que ne s'ajustent plus aux réalités actuelles. Probablement qu'au moment du surgissement de ces idées, elles correspondaient à quelque chose de fondamental dans la conservation de la vie d'une communauté ou répondaient à une problématique particulière. Je ne juge pas le passé. Mais, dans le présent, j'ose dire qu'une attitude fondamentaliste au niveau de certaines idées est capable d'introduire la mort à l'intérieur du désir de construction de la vie. Je prends comme exemple de cette pollution le développement de l'idée de résurrection dans le christianisme. Comme le dit saint Paul, «si le Christ n'a pas ressuscité, notre foi est vaine ». Mais, qui est le Christ? La tradition chrétienne a identifié le Christ à Jésus de Nazareth, le fils de Marie, celui qui a été conçu par l'Esprit saint. Or, ce Christ a pourtant une figure historique masculine, c'est-à-dire qu'il est identifié à un homme. En plus, dans l'iconographie chrétienne, il est toujours blanc avec des traits européens. 
Ces images sont toujours là et nous n'avons pas su introduire une autre théologie inclusive, suffisamment compréhensible et capable d'aider à créer une autre iconographie. Cela est déjà un symptôme qui signifie que les idées religieuses du passé demeurent dans l'art populaire et dans l'inconscient chrétien collectif.

La résistance aux changements est forte dans le régime des croyances religieuses et il nous faut du temps et de nombreux défis pour les faire changer. L'expérience de la résurrection est consacrée comme une expérience masculine, même si récemment on admet que les femmes ont été les premières à rendre témoignage et à annoncer la résurrection. Mais elles annoncent encore la résurrection de Jésus, du Seigneur, de l'unique Fils de Dieu. C'est en lui comme ressuscité que nous ressuscitons, femmes et hommes. Nous n'avons pas encore osé parler de résurrection autrement que dans les limites anthropocentriques et masculines. Et sans nous en rendre compte, par nos idées théologiques, nous polluons le monde et nous permettons que cette pollution continue d'agir dans l'inconscient collectif des gens. Il y a tout un travail éducatif et d'éveil de la conscience à faire dans nos différentes cultures pour « resignifier» nos valeurs et nos croyances.

Dépolluer un concept, c'est permettre qu'il se reconstruise à partir de nous, de notre vie au présent, de nos questions actuelles et, finalement, lui permettre de nous donner vie. Néanmoins, il me semble que la tradition théologique officielle est aveugle et sourde aux questions de la vie quotidienne des gens et aux défis lancés aux concepts théologiques. Les représentants de l'officialité continuent à donner leur parole sur les événements, parole d'autorité comme si elle venait de Dieu comme être suprême. Comment être au rendez-vous de l'histoire à partir de la poussière du chemin, à partir des larmes, du sang versé, des petites joies d'un repas en famille ou entre amis? Comment accueillir les changements sans nuire à l'histoire de notre humanité ?

Je reprends ici l'idée de la résurrection pour montrer les grandes lignes d'un essai de dépollution contextuelle de cette croyance. Ressusciter signifie revenir à la vie. C'est comme si, après une expérience de perte de vie, nous essayions de nous aider et d'être aidés à revenir à la vie, à un bien-être qui nous permet de vivre une certaine qualité de vie. C'est dans ce sens que le concept de résurrection touche à la vie d'abord, dans cette histoire même. Il s'agit des actions de résurrection, parce que nous sommes des êtres de mort et de vie dans la vie. Mort et vie sont aujourd'hui, maintenant. On croit que la vie est pour maintenant et la mort pour après, lorsque notre 
corps sera sans souffle. Sans doute qu'il y a de cela aussi. Mais la résurrection a d'abord à voir avec cette vie quotidienne, avec cet espace et ce temps qui sont les nôtres: la vie des sans domicile fixe, des aveugles, des paralysés, des prostitués, des violés, etc., et aussi la vie de la planète avec la multiplicité des êtres vivants. Tout vit, meurt et ressuscite... Une résurrection en même temps éthique et esthétique. Une résurrection au-delà de nos prévisions et de nos modèles. Nous ressuscitons en vivant la justice et la beauté. Nous ressuscitons par le pain partagé et par le beau coucher de soleil. Nous ressuscitons par la dépollution d'une rivière près de chez nous et nous ressuscitons quand notre fille revient à la maison ou quand on prend une boisson fraîche avec des amis. La résurrection devient un concept plus large et participe à une histoire et à une tradition plus vaste que celle du monde chrétien patriarcal. Dans cette perspective, nous croyons à la résurrection de Jésus et à celle de tant d'autres personnes puisqu'elles ont passé leur vie en essayant de faire vivre, c'est-à-dire en rendant la résurrection présente par l'affrontement des forces de destruction. C'est bien à cause de cela que la communauté chrétienne s'adresse à Jésus comme «le ressuscité », celui qui a vécu sa vie pour la vie.

Dépolluer un concept aujourd'hui, c'est lui permettre un contenu et un usage au-delà d'une vision trop patriarcale et anthropocentrique. Alors, la question "qui est mon prochain? » pourra être écoutée et vécue autrement.

Le salut cosmique ne pourra pas nous faire oublier notre prochain, de parler son langage, de le soulager dans ses douleurs immédiates. Les corps ne mentent pas... Ce sont eux qui dénoncent la nécessité de salut dans l'ensemble de la vie. Le réchauffement global n'est pas un problème à part des guerres, de la violence sans fin, de la production des armes, de la violation des corps d'enfants et de femmes. Tout est un tout, mais il faut commencer par mon semblable, mon prochain, mon image, mon village. Le mouvement contraire est aussi possible, mais il risque de nous éloigner de chez nous, de notre quartier, de notre voisin, de notre proche qui nous interpelle et nous dérange sans cesse.

\section{Pour conclure et continuer à échanger}

Le titre de cette réflexion qui arrive à sa fin — «La théologie au Brésil et le mouvement altermondialiste» —, très probablement, n'a pas reproduit la majorité des couleurs théologiques présentes dans cet immense pays. Je me rends compte que je n'ai pas fait un travail d'historienne pour exprimer 
la diversité présente. Ce n'est pas ma spécialité. Je viens d'achever un travail de réflexion à partir du Brésil, mon pays. J'ai livré un point de vue sans doute très limité et je vous invite à en chercher d'autres. Je n'ai pas écrit sur la théologie au Brésil, mais sur quelques aspects de la théologie vus par une femme brésilienne ou une théologienne au Brésil engagée dans certaines luttes pour la dignité humaine et la planète.

J'aimerais terminer ce texte en disant que les discours de la science et de la théologie académique doivent reprendre leur objectif d'œuvrer pour le bien des groupes humains dans la vie courante. Cette affirmation peut paraître d'une évidence dispensable dans cette conclusion. Mais je l'affirme quand même, comme un point de repère important pour moi-même et pour d'autres. Souvent nous pouvons nous noyer dans la beauté de nos constructions mentales, même au niveau des relectures théologiques et des interprétations bibliques, et cela ne se transmet pas aux communautés chrétiennes. Nous pouvons nous laisser éblouir par la beauté de nos théories et par leur capacité à nous faire vivre, en imagination, ce que nous désirons, mais les responsables des Églises n'en sont pas informés. Je nous invite à laisser entrer dans nos pensées la vie des gens et à lutter pour que des structures ecclésiales ouvrent des espaces pour penser autrement notre héritage de foi. L'imaginaire est dans le réel, mais le réel — c'est-à-dire l'aujourd'hui - a une grandeur spécifique qui ne peut pas être oubliée. C'est aujourd'hui qu'une femme a été battue, qu'un jeune a été tué, qu'un enfant pleure de faim, qu'un tremblement de terre se produit et que des morts sont entassés sur la place publique. C'est aujourd'hui qu'il faut dire une parole de consolation, faire un geste de tendresse, de partage de pain... C'est aujourd'hui qu'il faut aller aux tribunaux pour proclamer l'urgence de la justice... C'est aujourd'hui le jour de notre vie. Et c'est dans cet aujourd'hui joyeux ou dramatique que nous sommes invités à nous convertir au réel, c'est-à-dire aux visages des gens tels qu'ils sont, à essayer de reprendre et continuer l'aventure humaine toujours en pensant que l'autre est aussi moi dans l'autre. La théologie n'est que l'effort pour mettre un peu de lumière dans nos ténèbres, un peu de sel dans notre nourriture, un peu d'eau fraîche dans nos cœurs assoiffés. La théologie n'est que célébration de notre vie avec ses tristesses, joies et espérances. Finalement, c'est cela la théologie au quotidien. Elle ne s'oppose pas à la recherche scientifique de penser la foi et nos croyances religieuses, mais elle est plus que l'effort de renouvellement de nos concepts. Et c'est ce simple plus qui reste le défi continuel pour renouveler notre amour et notre foi aujourd'hui. 


\section{Références}

Arendt, H. (2005), Entre o passado e o futuro, São Paulo, Perspectiva.

Boff, L. (2001), Cuidar La tierra. Hacia una ética universal, México, Dabar.

Brighenti, A. (2009), "Gritos da Amazônia. A propósito do III Fórum Mundial de teologia e Libertação ", Revista Eclesiástica Brasileira, 275, p. 596-617.

Haught, J. (2009), "La science et la quête de la finalité cosmique ", Revue Théologique de Louvain, 40/4, p. 466-479.

Hervieu-Leger, D. (1999), Le pèlerin et le converti. La religion en mouvement, Paris, Flammarion.

Hunt, M. (2009), «Bodies don’t Lie: A Feminist Theological Perspective on Embodiment », Conférence donnée le 24 janvier, Belém (Brésil).

Susin, L. C. (2009), Mysterium Creationis. Um olhar interdisciplinar sobre o Universo, São Paulo, Soter/Paulinas.

\section{Résumé}

Cet article présente de façon critique quelques réflexions sur le mouvement altermondialiste et la théologie au Brésil. Débutant par un bref rappel historique sur le changement du rôle des religions et des théologies, l'auteur présente les théologies comme des pouvoirs qui veulent influencer les comportements des citoyens et des citoyennes. Néanmoins, ces pouvoirs sont plus complexes que du temps des utopies libertaires des années 1960-1990, notamment parce que des intérêts politiques, économiques et idéologiques se dissimulent souvent sous le voile de la religion. La théologie féministe est aussi présentée comme un pouvoir souhaitant changer les rapports inégaux qui caractérisent les institutions culturelles, ainsi que les représentations religieuses qui privilégient un christianisme à l'aspect masculin. L'article rappelle enfin combien les nouvelles thématiques de la théologie au Brésil sont marquées par les problèmes actuels des sciences sociales et de l'écologie, au risque d'oublier à nouveau la situation des femmes, souvent considérées comme des «sujets» subordonnés à d'autres. 


\section{Abstract}

This article presents in a critical way some reflections about the altermondialism movement and theology in Brazil. Starting with a brief historical review concerning some shifts in religions' and theologies' roles, the author presents theologies as powers trying to influence citizens' behavior. Nevertheless, those powers are more complex than they were in the age of libertarian utopias in the 1960s-1990s, especially because some political, economical, and ideological interests are draped in a religious veil. Feminist theology is also presented as a power trying to change the unfair treatments that characterize cultural institutions, as well as religious representations that favor a male-shaped Christianity. The paper then reminds the reader how new theological questions in Brazil are characterized by current problems in social sciences and ecology, which runs the risk of forgetting again the situation of women, often considered as «subjects» subordinated to others. 